\title{
A Broad-Band Power Spectrum Analyzer Based on Twin-Channel Delayed Sampling
}

\author{
Domenico Mirri, Member, IEEE, Gaetano Iuculano, Gaetano Pasini, Member, IEEE, \\ Fabio Filicori, Member, IEEE, and Lorenzo Peretto, Member, IEEE
}

\begin{abstract}
This paper describes a power spectrum analyzer whose bandwidth is not limited by the mean sampling time. The procedure is based on the estimation of the spectral components of the autocorrelation function of the input signal through the simultaneous random sampling of the given input signal and its randomly "delayed copy." The samples are therefore randomly taken in a double-dimension space, time, and delay. By using a random process in the time domain with a recursive mean previously introduced by the authors in order to avoid any bandwidth limitation due to the sampling strategy, it is shown both theoretically and through simulation that the estimate of the power spectral components is asymptotically unbiased on the unique hypothesis of a synchronized random sampling in the delay domain, i.e., the sampling delays are uniformly distributed in an interval equal to the period of the input signal. The simulation results confirm the theoretical findings.
\end{abstract}

Index Terms - Autocorrelation, performance analysis, power spectrum, random sampling.

\section{INTRODUCTION}

$\mathbf{T}$ HE potential advantages of particular random type sampling strategies in the implementation of broadband digital wattmeters have already been pointed out [1], [2]. In fact, we introduced two sampling techniques. One is described by the following additive model which defines a discrete parameter continuous random process with a recursive mean $T_{S}:$

$$
t_{w-i}=t_{0}+\left(w-i+Y_{w-i}\right) T_{S}
$$

where $w$ marks a sequence of sampling instants and $i$ a generic value of the sequence, $t_{w-i}$ is a sampling instant, $\tau_{0}$ the initial shift, $T_{S}$ a constant, $Y_{w-i}$ a random variable uniformly distributed in the interval $(0,1)$. The other is described by this additive model which defines a recursive random process

$$
t_{w-i}=t_{w-i-1}+\left(1+Y_{w-i}^{\prime}\right) T_{S}
$$

where $Y_{w-i}^{\prime}$ is a random variable uniformly distributed in the interval $(0, \approx 1.5)$. We theoretically demonstrated and experimentally verified that these sampling strategies with

Manuscript received September 29, 1995; revised November 30, 1998.

D. Mirri, G. Pasini, and L. Peretto are with the Dipartimento di Ingegneria Elettrica, Facoltà di Ingegneria, Bologna 40136 Italy.

G. Iuculano is with the Dipartimento di Elettronica, Facoltà di Ingegneria, Firenze 50125 Italy.

F. Filicori is with the Dipartimento di Elettronica, Facoltà di Ingegneria, Bologna 40136 Italy.

Publisher Item Identifier S 0018-9456(98)09840-4. the associated filtering algorithms do not introduce any bandwidth limitation when used to deduce the mean power. The bandwidth limitation is in this case due uniquely to the bandwidth of the sample and hold $(\mathrm{S} / \mathrm{H})$ device. In spectral analysis the quantities to be measured are, however, not only related to the set of sampled signal values (as in the case of the wattmeters), but also to their time allocation. To this end, a finely controllable sampling time spacing has been introduced in a basically "random" sampling strategy. In this way a simultaneous sampling of both the given input signal $x(t)$ and its "delayed copy" $x(t-\tau)$ can be carried out and the autocorrelation function of the signal can be estimated. This procedure, which allows us to evaluate the power spectrum of the input signal, is described in Section II. In Section III, the criterion for the performance analysis is carried out together with the theoretical findings, while in Section IV the simulation results are given and discussed.

\section{Measurement Method}

Let us consider the Fourier series of a finite spectrum periodic signal $x(t)$ with period $T_{1}=1 / f_{1}$

$$
x(t)=\sum_{k=-M}^{+M} X_{k} e^{j 2 \pi k f_{1} t}
$$

with $k$ integer, where the spectral coefficients $X_{k}=\left|X_{k}\right| e^{j \varphi_{k}}$ can be derived from

$$
X_{k}=\frac{1}{T_{1}} \int_{-T_{1} / 2}^{+T_{1} / 2} x(t) e^{-j 2 \pi k f_{1} t} d t
$$

When the signal is real, i.e., $x(t)=x^{*}(t)$, we can write $X_{-k}=X_{k}^{*}$ because the conjugate of an integral is the integral of the conjugate due to the additive property of the integral. By recalling the frequency shifting property [3], the Fourier transform of the signal $x(t)$ can be expressed as follows:

$$
X(f)=\sum_{k=-M}^{+M} X_{k} \delta\left(f-k f_{1}\right)
$$

and its inverse Fourier transform therefore becomes

$$
x(t)=\int_{-\infty}^{+\infty} X(f) e^{j 2 \pi f t} d f .
$$


The autocorrelation function of the real signal $x(t)$ is given by [4]

$$
\begin{aligned}
R(\tau) & =\langle x(t) x(t-\tau)\rangle \\
& =\frac{1}{T_{1}} \int_{-T_{1} / 2}^{+T_{1} / 2} x(t) x(t-\tau) d t \\
& =\sum_{k=-M}^{+M}\left|X_{k}\right|^{2} e^{j 2 \pi k f_{1} \tau} \\
& =\sum_{k=-M}^{+M}\left|X_{k}\right|^{2} \cos \left(2 \pi k f_{1} \tau\right)
\end{aligned}
$$

and it still contains all the information concerning the signal power spectrum components, i.e., $\left|X_{k}\right|^{2}$. The mean value is identified by the symbol $\langle\cdot\rangle$. The frequency allocation of each spectral component of $x(t)$ and $R(\tau)$ is the same; besides the phase angle of each spectral component of $x(t)$ disappears in the evaluation of $R(\tau)$, which therefore results in a real even function of $\tau$. For $\tau=0$ we obtain the well-known Parseval formula

$$
R(0)=\left\langle x^{2}(t)\right\rangle=\sum_{k=-M}^{+M}\left|X_{k}\right|^{2}
$$

which represents the mean power associated to the input signal $x(t)$.

Because also the autocorrelation function is a discrete spectrum signal with period $T_{1}$, we can write

$$
\begin{aligned}
\left|X_{k}\right|^{2} & =\frac{1}{T_{1}} \int_{-T_{1} / 2}^{+T_{1} / 2} R(\tau) e^{-j 2 \pi k f_{1} \tau} d \tau \\
& =\frac{1}{T_{1}} \int_{-T_{1} / 2}^{+T_{1} / 2} R(\tau) \cos \left(2 \pi k f_{1} \tau\right) d \tau
\end{aligned}
$$

Due to the frequency shifting property [3], the Fourier transform of the autocorrelation function (7), i.e., the power density spectrum $S(f)$ of the signal $x(t)$, can be expressed as follows:

$$
S(f)=\sum_{k=-M}^{+M}\left|X_{k}\right|^{2} \delta\left(f-k f_{1}\right)
$$

where also $S(f)$ is a real nonnegative and even function. This function measures the distribution of power in $x(t)$ as a function of frequency [4]. The autocorrelation function, representing the inverse Fourier transform of the power density spectrum, can be expressed as follows:

$$
R(\tau)=\int_{-\infty}^{+\infty} S(f) e^{j 2 \pi f \tau} d f
$$

Since

$$
R(0)=\left\langle x^{2}(t)\right\rangle=\int_{-\infty}^{+\infty} S(f) d f
$$

we can write

$$
\begin{aligned}
|R(\tau)| & =\left|\int_{-\infty}^{+\infty} S(f) e^{j 2 \pi f \tau} d f\right| \\
& \leq \int_{-\infty}^{+\infty}\left|S(f) e^{j 2 \pi f \tau}\right| d f \\
& =\int_{-\infty}^{+\infty} S(f) d f=R(0) .
\end{aligned}
$$

Therefore the autocorrelation function, periodic with the same period $T_{1}$ of the signal, has its largest magnitude at periodic intervals $\tau=0, \pm T_{1}, \pm 2 T_{1}, \ldots$.

By substituting (7) into (9) we obtain

$\left|X_{k}\right|^{2}=\frac{1}{T_{1}^{2}} \int_{-T_{1} / 2}^{+T_{1} / 2} \int_{-T_{1} / 2}^{+T_{1} / 2} x(t) x(t-\tau) \cos \left(2 \pi k f_{1} \tau\right) d t d \tau$

This double integral in the time and delay domain can be numerically computed as the average of a number $N$ of samples randomly taken in the double-dimension $(t, \tau)$ space by using the following formula where the integer $w$ marks a generic value of the output:

$$
\begin{aligned}
&\left|\tilde{X}_{k, w}\right|^{2}= \frac{1}{N} \sum_{i=0}^{N-1} x\left(t_{w-i}\right) x\left(t_{w-i}-\tau_{w, i}\right) \cos \left(2 \pi k f_{1} \tau_{w i}\right) \\
&= \frac{1}{N} \sum_{i=0}^{N-1} x\left(t_{w-i}\right) x\left(t_{w-i}-\tau_{w i}\right) \\
& \cdot\left[\frac{e^{j 2 \pi k f_{1} \tau_{w i}}+e^{-j 2 \pi k f_{1} \tau_{w i}}}{2}\right] \\
& \text { for } k=0,1, \cdots, M
\end{aligned}
$$

according to a Monte Carlo-like statistical approach for integral evaluation [5]. In particular, each $i$ th sampling point in the $(t, \tau)$ space is randomly generated according to the sampling strategy defined by (1) and the following formula for the delay:

$$
\tau_{w i}=Z_{w i} T_{A}
$$

being $\tau_{w i}$ the $i$ th random delay used to deduce the $w$ th output estimate, $T_{A}$ a generic value, and $Z_{w i}$ independent random variables uniformly distributed in the interval 0 to 1 . The properties of this approach are outlined in the following section.

\section{Performance AnAlysis}

The estimated $k$ th power spectral component of the input signal through (15) is a function of the variables $t_{0}, w$ and of the vectors of the two independent, uniformly distributed, random variables $\underline{Y}_{w}=\left\{Y_{w-i}\right\}, \underline{Z}_{w}=\left\{Z_{w i}\right\}$ used to obtain the output labeled with $w$, i.e., $\left|\tilde{X}_{k w}\right|^{2}=f\left(t_{0}, w, \underline{Y}_{w}, \underline{Z}_{w}\right)$. The labeling mark $w$ identifies a generic discrete output of the instrument for each $k$ th spectral component; therefore the integer $w$ is random in nature because it can be considered randomly picked up from a sequence of $2 h+1$ successive potential measurement occasions $(-h \leq k \leq+h)$, each of which has an equal chance of being selected. The value of $t_{0}$ is not known a priori and one is led to interpret any value of $t_{0}$ as an outcome of a random variable with a continuous set of values uniformly distributed in some generic time interval $T$ which encompasses all the possible realizations $(-T / 2 \leq$ $t_{0} \leq+T / 2, T$ being unknown). Therefore, we introduce $t_{0}$ as a continuous random variable uniformly distributed in the time interval $T$ and $w$ as a discrete one uniformly distributed in the interval $2 h+1$. On the hypothesis of a random sampling strategy defined according to (1), the independent random variables of the vector $\underline{Y}_{w}$ are uniformly distributed in the 
interval 0 to 1 ; from (16) we deduce that also the independent random variables of the vector $\underline{Z}_{w}$ are uniformly distributed in the same interval.

An appropriate characterization of the output uncertainty can be obtained by evaluating the statistical parameters of $\left|\tilde{X}_{k w}\right|^{2}$, i.e., the mean value $M\left\{\left|\tilde{X}_{k w}\right|^{2}\right\}$ and the mean square error $E^{2}=M\left\{\left(\left|\tilde{X}_{k w}\right|^{2}-\left|X_{k}\right|^{2}\right)^{2}\right\}$. In order to incorporate all the $a$ priori chances into the instrument's performance, the number of the output states, i.e., $2 h+1$, must be sufficiently large and theoretically tend to infinite. Therefore we must consider the asymptotic statistical parameters of the output, i.e., the asymptotic mean $M\left\{\left|\tilde{X}_{k w}\right|^{2}\right\}$ and the asymptotic mean square error (mse) $E^{2}=M\left\{\left(\left|\tilde{X}_{k w}\right|^{2}-\left|X_{k}\right|^{2}\right)^{2}\right\}$.

It can be shown (see the Appendix) that, on the hypothesis of a delay $\tau_{w i}$ uniformly distributed in a time interval equal to the period $T_{1}$ of the input signal, the asymptotic bias is null (A14)

$$
\underset{M}{M}\left\{\left|\tilde{X}_{k w}\right|^{2}\right\}=\left|X_{k}\right|^{2}
$$

Therefore the asymptotic mean square error coincides with the asymptotic variance, which can be expressed as follows (A31):

$$
\begin{aligned}
\operatorname{Var}\left\{\left|\tilde{X}_{k, w}\right|^{2}\right\} & \frac{1}{2 N}\left[\left(\sum_{r=-M}^{+M}\left|X_{r}\right|^{2}\right)^{2}+\left|\sum_{r=-M}^{+M} X_{r} X_{2 k-r}\right|^{2}\right] \\
& +\frac{1}{2} \sum_{r=-M}^{+M}\left\{\operatorname{Re}\left[X_{r} X_{k}^{2} X_{2 k+r}^{*}\right]+\left|X_{r}\right|^{2}\left|X_{k}\right|^{2}\right\} \\
& \cdot \operatorname{sinc}^{2}\left((r+k) f_{1} T_{S}\right)\left(\frac{\operatorname{sinc}^{2}\left((r+k) N f_{1} T_{S}\right)}{\operatorname{sinc}^{2}\left((r+k) f_{1} T_{S}\right)}-\frac{1}{N}\right) \\
& -\left|X_{k}\right|^{4} .
\end{aligned}
$$

On the hypothesis that $f_{1} T_{s}=r$ with $r$ an integer (i.e., a synchronous sampling strategy) we obtain

$$
\begin{aligned}
\operatorname{Var}\left\{\left|\tilde{X}_{k, w}\right|^{2}\right\} & =\frac{1}{2 N} \sum_{r=-M}^{+M}\left|X_{r}\right|^{2} \sum_{u=-M}^{+M}\left|X_{u}\right|^{2} \\
& =\frac{1}{2 N}\left(\sum_{r=-M}^{+M}\left|X_{r}\right|^{2}\right)^{2} .
\end{aligned}
$$

\section{Simulation Results}

A power spectrum analyzer based on the operating principle defined by (15) was simulated by assuming $T_{s} \approx 100 \mu \mathrm{s}$; each spectral component was estimated by considering $N$ random samplings of the input signal. It is important to observe that the results were essentially the same independent of the random sampling strategy adopted (1) or (2). Table I shows the asymptotic bias and standard error of a periodic sinusoidal input signal with an amplitude equal to $2 \mathrm{~V}$ at different frequencies from $1 \mathrm{kHz}$ to $1 \mathrm{GHz}$ estimated by considering $N=10^{2}$ samples. In order to evaluate the asymptotic bias and standard error, each measurement simulation was repeated $10^{3}$ and $10^{4}$ times. These results are not, in practice, frequency
TABLE I

Bias and Standard ERror (Theoretical and Simulated) of the Simulated Measurements for Sinusoidal InPut Signals at Different Frequencies BetweEn $1 \mathrm{kHz}$ and $1 \mathrm{GHz}$

\begin{tabular}{c|c|c|c|c|c|c}
\hline & \multicolumn{4}{|c|}{$N_{R}=10^{3}$} & \multicolumn{3}{c}{$N_{R}=10^{4}$} \\
\hline Frequency $(\mathrm{MHz})$ & Bias & $\begin{array}{c}\text { Theoretical } \\
\text { std. crror }\end{array}$ & $\begin{array}{c}\text { Simulated } \\
\text { sid. error }\end{array}$ & Bias & $\begin{array}{c}\text { Theoretical } \\
\text { std. error }\end{array}$ & $\begin{array}{c}\text { Simulated } \\
\text { std. error }\end{array}$ \\
\hline $1 \mathrm{kHz}$ & $-3.8 \cdot 10^{4}$ & $3.3 \cdot 10^{-3}$ & $3.2 \cdot 10^{-7}$ & $1.8 \cdot 10^{-3}$ & $1.0 \cdot 10^{-3}$ & $1.0 \cdot 10^{-3}$ \\
\hline 10 & $-5.8 \cdot 10^{-3}$ & $3.9 \cdot 10^{-3}$ & $3.8 \cdot 10^{-3}$ & $2.6 \cdot 10^{-4}$ & $1.2 \cdot 10^{-3}$ & $1.2 \cdot 10^{-3}$ \\
\hline 100 & $-2.1 \cdot 10^{5}$ & $3.9 \cdot 10^{-3}$ & $3.9 \cdot 10^{-3}$ & $1.8 \cdot 10^{-3}$ & $1.2 \cdot 10^{-3}$ & $1.2 \cdot 10^{-3}$ \\
\hline $1 \mathrm{MIz}$ & $-2.3 \cdot 10^{-3}$ & $3.9 \cdot 10^{-3}$ & $3.7 \cdot 10^{-3}$ & $-6.7 \cdot 10^{-5}$ & $1.2 \cdot 10^{-3}$ & $1.2 \cdot 10^{3}$ \\
\hline 10 & $2.6 \cdot 10^{-3}$ & $3.9 \cdot 10^{3}$ & $3.7 \cdot 10^{-3}$ & $1.0 \cdot 10^{-3}$ & $1.2 \cdot 10^{3}$ & $1.2 \cdot 10^{-3}$ \\
\hline 100 & $7.4 \cdot 10^{4}$ & $3.9 \cdot 10^{3}$ & $3.9 \cdot 10^{-3}$ & $-6.0 \cdot 10^{-4}$ & $1.2 \cdot 10^{-3}$ & $1.2 \cdot 10^{-3}$ \\
\hline $1 \mathrm{GHz}$ & $-1.9 \cdot 10^{-3}$ & $3.9 \cdot 10^{-3}$ & $3.7 \cdot 10^{-3}$ & $5.2 \cdot 10^{-5}$ & $1.2 \cdot 10^{-3}$ & $1.2 \cdot 10^{-3}$ \\
\hline
\end{tabular}

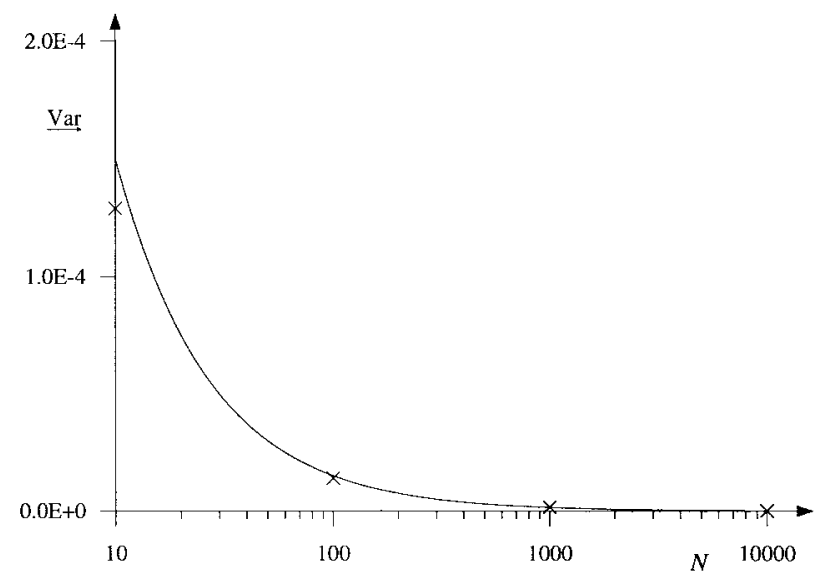

Fig. 1. Estimate of the asymptotic variance Var as a function of the number of the samples for each measurement.

dependent. A very small (theoretically zero) bias is confirmed by observing that the error associated to "measured" values (i.e., simulated) are within the expected range correspondent to three times the standard error.

Fig. 1 shows the shape of the asymptotic variance as a function of the number $N$ of sampling values used to estimate the spectral component of a periodic sinusoidal signal with an amplitude of $1 \mathrm{~V}$ and $f=1 \mathrm{kHz}$; the continuous curve represents the corresponding theoretical values for the asymptotic variance according to (18). Clearly, simulated and theoretically quantities are in very good agreement.

In Fig. 2(a) theoretical and simulated results are compared in the case of the power spectrum measurement, with $N=$ $N_{r}=10^{3}$, of a $1-\mathrm{V}$ sinusoidal signal with frequency variable from $1 \mathrm{kHz}$ to $1 \mathrm{GHz}$; Fig. 2(b) and (c) shows, respectively, the corresponding values for the bias and the variance.

Fig. 3(a) shows in a semilogarithmic scale the theoretical and simulated power spectral components of a square-wave together with the correspondent value of bias. It is interesting to observe that, according to the theory, the variance relative to the dc component is approximately twice that of the other components.

\section{CONCLUSIONS}

A power spectrum analyzer based on the estimation of the spectral components of the autocorrelation function of 


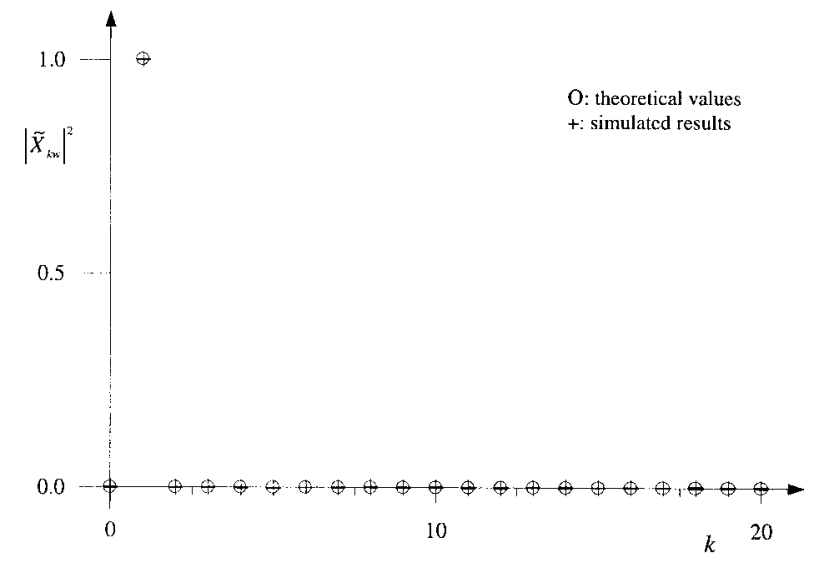

(a)

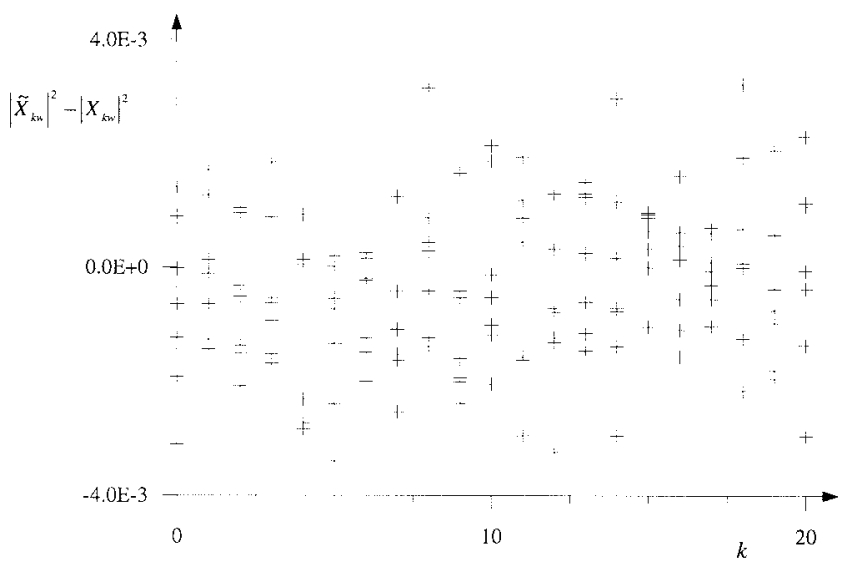

(b)

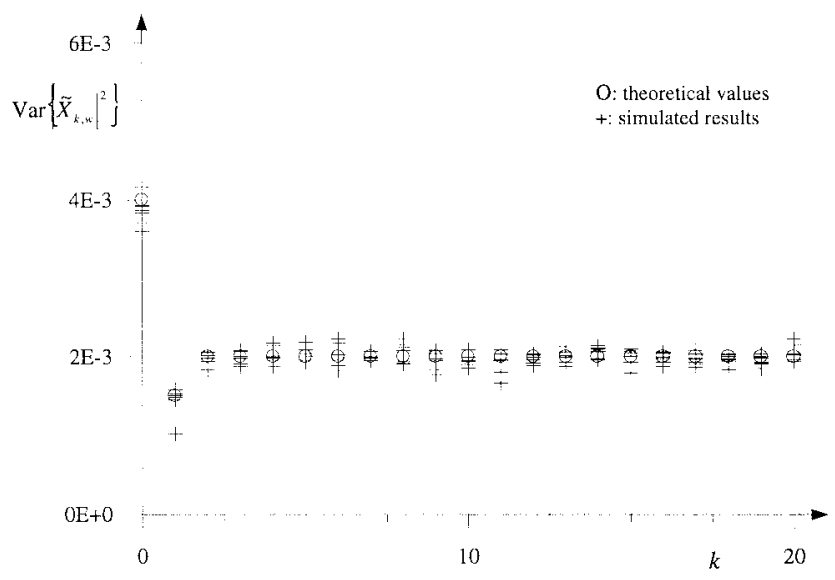

(c)

Fig. 2. Comparison between theoretical and simulated results in the case of the power spectrum measurement, with $N=N_{r}=10^{3}$, of a $1-\mathrm{V}$ sinusoidal signal with (a) frequency variable from $1 \mathrm{kHz}$ to $1 \mathrm{GHz}$, (b) corresponding values for bias, and (c) variance.

the input signal through the simultaneous random sampling of the given input signal and its randomly "delayed copy" was introduced. Therefore the samples are randomly taken in a double-dimension space, time, and delay. In order to evaluate the performance of the proposed method irrespective of each measurement occasion, the asymptotic statistical properties of the output must be considered. It was shown that the asymptotic bias is null and the expression of the

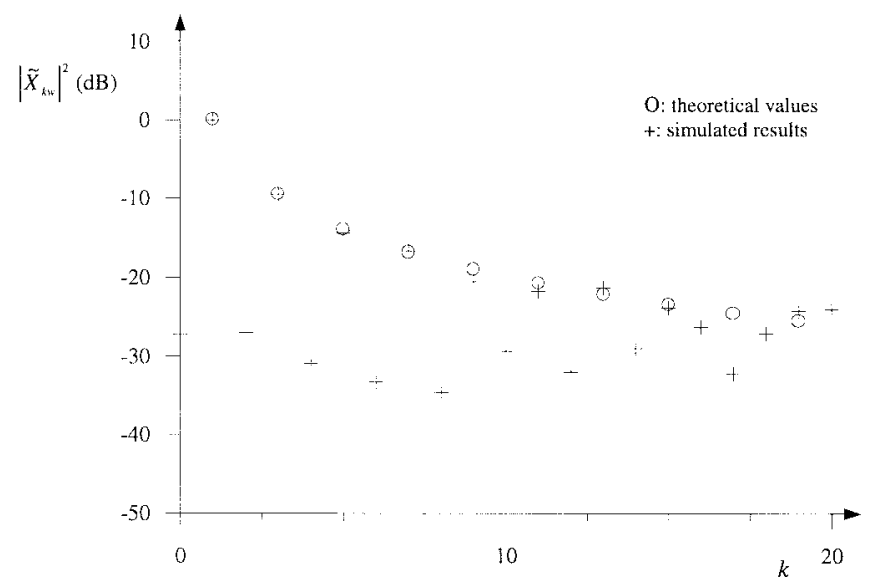

(a)

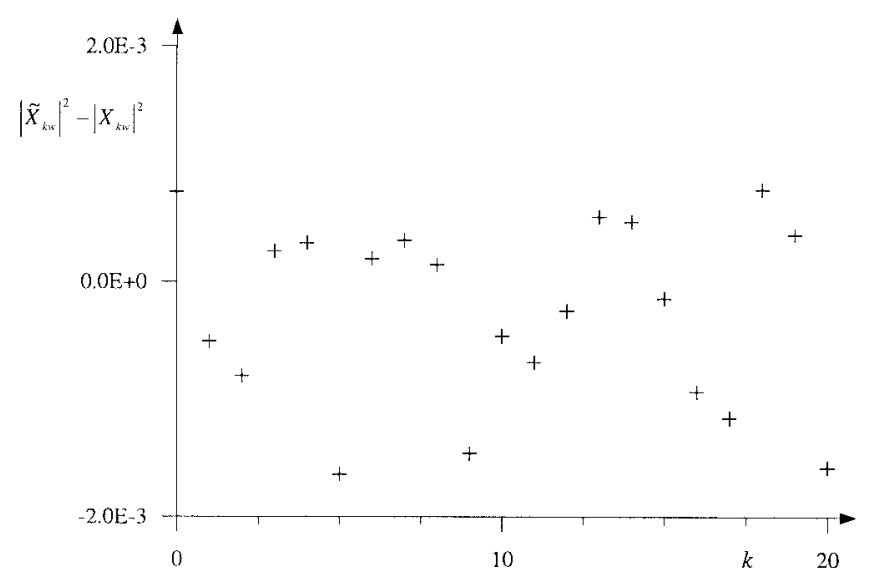

(b)

Fig. 3. Theoretical and simulated power spectral components of a square-wave (a) in a semilogarithmic scale and (b) together with the correspondent value of bias.

asymptotic variance was deduced on the unique hypothesis of a synchronized random sampling in the delay domain, i.e., the sampling delays are uniformly distributed in an interval equal to the period of the input signal. By using a random process in the time domain with a recursive mean or a recursive random process previously introduced by the authors in order to avoid any bandwidth limitation due to the sampling strategy, the theoretical findings were in very good accordance with the simulated ones.

\section{APPENDIX}

By substituting (1) and (3) into (15) and taking into account (16), we obtain

$$
\begin{aligned}
\left|\tilde{X}_{k, w}\right|^{2}= & \frac{1}{N} \sum_{r=-M}^{+M} \sum_{u=-M}^{+M} X_{r} X_{u} e^{j 2 \pi(r+u) f_{1}\left(t_{0}+w T_{S}\right)} \\
& \cdot \sum_{i=0}^{N-1} e^{j 2 \pi(r+u)\left(-i+Y_{w-i}\right) f_{1} T_{S}} \\
& \cdot\left[\frac{e^{-j 2 \pi(u-k) Z_{w i} f_{1} T_{A}}+e^{-j 2 \pi(u+k) Z_{w i} f_{1} T_{A}}}{2}\right] .
\end{aligned}
$$


For the evaluation of the statistical mean of this quantity it is necessary to consider the simultaneous random contributions of the variables $w, t_{0}$, and of the vectors $\underline{Y}_{w}=\left\{Y_{w-i}\right\}, \underline{Z}_{w}=$ $\left\{Z_{w i}\right\}$. To this end it is convenient to recall the following relationships. By considering the characteristic function of a continuous random variable $Y$ uniformly distributed in the interval $0-1$, we obtain [6]

$$
\begin{aligned}
M\left\{e^{j 2 \pi \alpha Y}\right\} & =\int_{0}^{1} f(y) e^{j 2 \pi \alpha y} d y \\
& =\frac{e^{j 2 \pi \alpha}-1}{j 2 \pi \alpha} \\
& =e^{j \pi \frac{\sin (\pi \alpha)}{\pi \alpha}} \\
& =e^{j \pi} \operatorname{sinc}(\alpha)
\end{aligned}
$$

where $\alpha$ is a real number and $f(y)=1$ is the uniform probability density. Further, a continuous random variable $t_{0}$ uniformly distributed in the interval $\pm T / 2$ has the following characteristic function:

$$
\begin{aligned}
M\left\{e^{j 2 \pi \alpha t_{0}}\right\} & =\int_{-T / 2}^{+T / 2} f(t) e^{j 2 \pi \alpha t} d t \\
& =\frac{1}{T} \frac{e^{j \pi \alpha T}-e^{-j \pi \alpha T}}{j 2 \pi \alpha} \\
& =\frac{\sin (\pi \alpha T)}{\pi \alpha T} \\
& =\operatorname{sinc}(\alpha T)
\end{aligned}
$$

where $f(t)=1 / T$ is the uniform probability density. The characteristic function of a discrete random variable $w$ uniformly distributed in the interval $\pm h$ can be expressed taking into account the properties of the geometric progressions with
$(2 h+1)$ terms as follows:

$$
\begin{aligned}
M\left\{e^{j 2 \pi \alpha w}\right\} & =\sum_{k=-h}^{+h} \operatorname{Prob}\{w=k\} e^{j 2 \pi \alpha k} \\
& =\frac{1}{2 h+1} \sum_{k=-h}^{+h} e^{j 2 \pi \alpha k} \\
& =\frac{1}{2 h+1} \frac{e^{j 2 \pi \alpha(h+1)}-e^{-j 2 \pi \alpha h}}{e^{j 2 \pi \alpha}-1} \\
& =\frac{1}{2 h+1} \frac{\sin (\pi \alpha(2 h+1))}{\sin (\pi \alpha)} \\
& =\frac{\operatorname{sinc}(\alpha(2 h+1))}{\operatorname{sinc}(\alpha)}
\end{aligned}
$$

where $\operatorname{Prob}\{w=k\}=1 /(2 h+1)$ is the uniform point probability of the discrete variable $w$. If $\alpha$ is an integer, the result of (A4) becomes identically equal to one.

Finally, the expression of a finite sum must be recalled

$$
\begin{aligned}
\sum_{i=0}^{N-1} e^{j 2 \pi \alpha i} & =\frac{e^{j 2 \pi \alpha N}-1}{e^{j 2 \pi \alpha}-1} \\
& =e^{j \pi \alpha(N-1)} \frac{\sin (\pi \alpha N)}{\sin (\pi \alpha)} \\
& =e^{j \pi \alpha(N-1)} N \frac{\operatorname{sinc}(\alpha N)}{\operatorname{sinc}(\alpha)}
\end{aligned}
$$

If $\alpha$ is an integer the sum in (A5) becomes identically equal to $N$.

Now, we can easily deduce the statistical mean value of $\left|\tilde{X}_{k, w}\right|^{2}$ from (A1). Taking into account the independence of all the random variables involved, from (A1) we deduce as shown in (A6) at the bottom of the page. By supposing each random delay $\tau_{w i}$ (16) uniformly distributed in a time interval equal to the period of the input signal $\left(T_{A}=T_{1}\right.$,

$$
\begin{aligned}
M\left\{\left|\tilde{X}_{k, w}\right|^{2}\right\}= & \frac{1}{N} \sum_{r=-M}^{+M} \sum_{u=-M}^{+M} X_{r} X_{u} M\left\{e^{j 2 \pi(r+u) f_{1} t_{0}}\right\} M\left\{e^{j 2 \pi(r+u) w f_{1} T_{S}}\right\} \sum_{i=0}^{N-1} e^{-j 2 \pi(r+u) i f_{1} T_{S}} \\
& \cdot M\left\{e^{j 2 \pi(r+u) Y_{w-i} f_{1} T_{S}}\right\}\left[\frac{M\left\{e^{-j 2 \pi(u-k) Z_{w i} f_{1} T_{A}}\right\}+M\left\{e^{-j 2 \pi(u+k) Z_{w i} f_{1} T_{A}}\right\}}{2}\right] \\
= & \left.\frac{1}{N} \sum_{r=-M} \sum_{u=-M}^{+M} X_{r} X_{u} \operatorname{sinc}\left((r+u) f_{1} T\right) \frac{\operatorname{sinc}\left((r+u)(2 h+1) f_{1} T_{S}\right)\left[\sum_{i=0}^{N-1} e^{-j 2 \pi(r+u) i f_{1} T_{S}}\right]}{\operatorname{sinc}\left((r+u) f_{1} T_{S}\right)}\right] \\
& \cdot e^{j \pi(r+u) f_{1} T_{S}} \operatorname{sinc}\left((r+u) f_{1} T_{S}\right)\left[\frac{e^{-j \pi(u-k) f_{1} T_{A}} \operatorname{sinc}\left((u-k) f_{1} T_{A}\right)+e^{-j \pi(u+k) f_{1} T_{A}} \operatorname{sinc}\left((u+k) f_{1} T_{A}\right)}{2}\right] \\
= & \sum_{r=-M} \sum_{u=-M}^{+M} X_{r} X_{u} \operatorname{sinc}\left((r+u) f_{1} T\right) \operatorname{sinc}\left((r+u)(2 h+1) f_{1} T_{S}\right) e^{-j \pi(r+u)(N-1) f_{1} T_{S}} \frac{\operatorname{sinc}\left((r+u) N f_{1} T_{S}\right)}{\operatorname{sinc}\left((r+u) f_{1} T_{S}\right)} \\
& \cdot e^{j \pi(r+u) f_{1} T_{S}}\left[\frac{e^{-j \pi(u-k) f_{1} T_{A}} \operatorname{sinc}\left((u-k) f_{1} T_{A}\right)+e^{-j \pi(u+k) f_{1} T_{A}} \operatorname{sinc}\left((u+k) f_{1} T_{A}\right)}{2}\right] .
\end{aligned}
$$


i.e., $f_{1} T_{A}=1$ ), i.e., the variability range of the time-delay is synchronized with the input signal, we have

$$
\operatorname{sinc}\left((u \pm k) f_{1} T_{A}\right)=\operatorname{sinc}(u \pm k)= \begin{cases}1, & \text { for } u=\mp k \\ 0, & \text { elsewhere }\end{cases}
$$

where $u$ and $k$ are integers. On the further hypothesis that also the mean sampling period $T_{S}$ is synchronous with the period $T_{1}$, i.e., $T_{S}=m T_{1}$ with $m$ a positive integer, by recalling that

$$
\begin{aligned}
\operatorname{sinc}\left((r+u) f_{1} T_{S}\right) & =\operatorname{sinc}(m(r+u)) \\
& = \begin{cases}1, & \text { for } r=-u \\
0, & \text { elsewhere }\end{cases}
\end{aligned}
$$

where $r$ and $u$ are integers, and that (A5)

$$
e^{-j \pi(r+u)(N-1) m} \frac{\operatorname{sinc}((r+u) N m)}{\operatorname{sinc}((r+u) m)}=1
$$

for any integer $m$, we can write

$$
M\left\{\left|\tilde{X}_{k w}\right|^{2}\right\}=\left|X_{k}\right|^{2}
$$

When the synchronous sampling satisfies instead the condition $T_{1}=m T_{S}$ with $m$ a positive integer, or the mean sampling pe$\operatorname{riod} T_{S}$ is asynchronous [remaining in any case synchronized the variability range of the delay $\tau_{w, i}$ and so (A7) is always verified], it must be considered in general the asymptotic behavior. To this end all the possible a priori chances of the output state, i.e., the number of the output states $(2 h+1)$, must be incorporated by considering $h$ sufficiently large and theoretically tending to infinity. Thus, in the following, we consider the asymptotic mean defined by

$$
\underset{\rightarrow}{M}\{\cdot\}=\lim _{h \rightarrow \infty} M\{\cdot\}
$$

no matter the value of $f_{1} T_{s}$. By referring to the first term of (A6) we can write (A11) as shown at the bottom of the page. By recalling that

$$
\begin{aligned}
\lim _{h \rightarrow \infty} \operatorname{sinc}((r & \left.+u)(2 h+1) f_{1} T_{S}\right) \\
& =\lim _{h \rightarrow \infty} \frac{\sin \left(\pi(r+u)(2 h+1) f_{1} T_{S}\right)}{\pi(r+u)(2 h+1) f_{1} T_{S}} \\
& = \begin{cases}1 & \text { for } r=-u \\
0 & \text { for } r \neq-u\end{cases}
\end{aligned}
$$

we obtain (A13) as shown at the bottom of the page. It is evident that, when the variability range of the delay $\tau_{w, i}$ is synchronized with the period $T_{1}$, by taking into account (A7), the nonnull contributions are only for $u= \pm k$ and therefore

$$
\underset{M}{M}\left\{\left|\tilde{X}_{k, w}\right|^{2}\right\}=\frac{\left|X_{k}\right|^{2}+\left|X_{-k}\right|^{2}}{2}=\left|X_{k}\right|^{2}
$$

Therefore, the asymptotic bias is in every case null.

Now we consider the asymptotic mean square error $E$ of $\left|\tilde{X}_{k w}\right|^{2}$ which coincides with its asymptotic variance since the asymptotic bias is null. In fact, we have

$$
\begin{aligned}
\stackrel{E^{2}}{\rightarrow} & \underset{\rightarrow}{\rightarrow}\left\{\left(\left|\tilde{X}_{k, w}\right|^{2}-\left|X_{k}\right|^{2}\right)^{2}\right\} \\
= & \underset{\rightarrow}{M}\left\{\left|\tilde{X}_{k, w}\right|^{4}\right\}-2\left|X_{k}\right|^{2} \underset{\rightarrow}{\rightarrow}\left\{\left|\tilde{X}_{k, w}\right|^{2}\right\}+\left|X_{k}\right|^{4} \\
= & \underset{\rightarrow}{M}\left\{\left|\tilde{X}_{k, w}\right|^{4}\right\}-\left|X_{k}\right|^{4} \\
= & \underset{\rightarrow}{\operatorname{Var}}\left\{\left|\tilde{X}_{k, w}\right|^{2}\right\}
\end{aligned}
$$

Thus, in order to evaluate the asymptotic variance of $\left|\tilde{X}_{k w}\right|^{2}$,

$$
\begin{aligned}
\underset{\rightarrow}{M}\left\{\left|\tilde{X}_{k, w}\right|^{2}\right\}= & \sum_{r=-M}^{r=+M} \sum_{u=-M}^{+M} X_{r} X_{u} \operatorname{sinc}\left((r+u) f_{1} T\right)\left[\lim _{h \rightarrow \infty} \operatorname{sinc}\left((r+u)(2 h+1) f_{1} T_{S}\right)\right] e^{-j \pi(r+u)(N-2) f_{1} T_{S}} \\
& \cdot \frac{\operatorname{sinc}\left((r+u) N f_{1} T_{S}\right)}{\operatorname{sinc}\left((r+u) f_{1} T_{S}\right)}\left[\frac{e^{-j \pi(u-k) f_{1} T_{A}} \operatorname{sinc}\left((u-k) f_{1} T_{A}\right)+e^{-j \pi(u+k) f_{1} T_{A}} \operatorname{sinc}\left((u+k) f_{1} T_{A}\right)}{2}\right] .
\end{aligned}
$$

$$
\underset{\rightarrow}{M}\left\{\left|\tilde{X}_{k, w}\right|^{2}\right\}=\sum_{u=-M}^{+M}\left|X_{u}\right|^{2}\left[\frac{e^{-j \pi(u-k) f_{1} T_{A}} \operatorname{sinc}\left((u-k) f_{1} T_{A}\right)+e^{-j \pi(u+k) f_{1} T_{A}} \operatorname{sinc}\left((u+k) f_{1} T_{A}\right)}{2}\right] .
$$


we must first deduce from (A1) the term $\left|\tilde{X}_{k, w}\right|^{4}$

$$
\begin{aligned}
& \left|\tilde{X}_{k, w}\right|^{4}=\frac{1}{N^{2}} \sum_{r=-M}^{+M} \sum_{u=-M}^{+M} \sum_{r^{\prime}=-M}^{+M} \sum_{u^{\prime}=-M}^{+M} X_{r} X_{u} \\
& \text { - } X_{r^{\prime}} X_{u^{\prime}} e^{j 2 \pi\left(r+u+r^{\prime}+u^{\prime}\right) f_{1}\left(t_{0}+w T_{S}\right)} \\
& \cdot \sum_{i=0}^{N-1} \sum_{i^{\prime}=0}^{N-1} e^{-j 2 \pi\left[(r+u) i+\left(r^{\prime}+u^{\prime}\right) i^{\prime}\right] f_{1} T_{S}} \\
& \text { - } e^{j 2 \pi\left[(r+u) Y_{w-i}+\left(r^{\prime}+u^{\prime}\right) Y_{w-i^{\prime}}\right] f_{1} T_{S}} \\
& \cdot\left[\frac{e^{-j 2 \pi\left[(u-k) Z_{w i}\right] f_{1} T_{A}}+e^{-j 2 \pi\left[(u+k) Z_{w i}\right] f_{1} T_{A}}}{2}\right] \\
& \cdot\left[\frac{e^{-j 2 \pi\left[\left(u^{\prime}-k\right) Z_{w i^{\prime}}\right] f_{1} T_{A}}+e^{-j 2 \pi\left[\left(u^{\prime}+k\right) Z_{w i^{\prime}}\right] f_{1} T_{A}}}{2}\right] \\
& =\frac{1}{4 N^{2}} \sum_{r=-M}^{+M} \sum_{u=-M}^{+M} \sum_{r^{\prime}=-M}^{+M} \sum_{u^{\prime}=-M}^{+M} X_{r} X_{u} \\
& \text { - } X_{r^{\prime}} X_{u^{\prime}} e^{j 2 \pi\left(r+u+r^{\prime}+u^{\prime}\right) f_{1}\left(t_{0}+w T_{S}\right)} \\
& \cdot \sum_{i=0}^{N-1} \sum_{i^{\prime}=0}^{N-1} e^{-j 2 \pi\left[(r+u) i+\left(r^{\prime}+u^{\prime}\right) i^{\prime}\right] f_{1} T_{S}} \\
& \text { - } e^{j 2 \pi\left[(r+u) Y_{w-i}+\left(r^{\prime}+u^{\prime}\right) Y_{w-i^{\prime}}\right] f_{1} T_{S}} \\
& \cdot\left[e^{-j 2 \pi\left[(u-k) Z_{w i}+\left(u^{\prime}-k\right) Z_{w i^{\prime}}\right] f_{1} T_{A}}\right. \\
& +e^{-j 2 \pi\left[(u-k) Z_{w i}+\left(u^{\prime}+k\right) Z_{w i^{\prime}}\right] f_{1} T_{A}} \\
& +e^{-j 2 \pi\left[(u+k) Z_{w i}+\left(u^{\prime}-k\right) Z_{w i}\right] f_{1} T_{A}} \\
& \left.+e^{-j 2 \pi\left[(u+k) Z_{w i}+\left(u^{\prime}+k\right) Z_{w i^{\prime}}\right] f_{1} T_{A}}\right] .
\end{aligned}
$$

Successively, its asymptotic mean value $M\left\{\left|\tilde{X}_{k, w}\right|^{4}\right\}$ can be deduced taking into account the independence of all the random variables involved

$$
\begin{aligned}
& \underset{\rightarrow}{M}\left\{\left|\tilde{X}_{k, w}\right|^{4}\right\}=\frac{1}{4 N^{2}} \sum_{r=-M}^{+M} \sum_{u=-M}^{+M} \sum_{r^{\prime}=-M}^{+M} \sum_{u^{\prime}=-M}^{+M} X_{r} X_{u} \\
& \text { - } X_{r^{\prime}} X_{u^{\prime}} M\left\{e^{j 2 \pi\left(r+u+r^{\prime}+u^{\prime}\right) f_{1} t_{0}}\right\} \\
& \cdot \underset{M}{M}\left\{e^{j 2 \pi\left(r+u+r^{\prime}+u^{\prime}\right) w f_{1} T_{S}}\right\} \\
& \cdot \sum_{i=0}^{N-1} \sum_{i^{\prime}=0}^{N-1} e^{-j 2 \pi\left[(r+u) i+\left(r^{\prime}+u^{\prime}\right) i^{\prime}\right] f_{1} T_{S}} \\
& \text { - } M\left\{e^{j 2 \pi\left[(r+u) Y_{w-i}+\left(r^{\prime}+u^{\prime}\right) Y_{w-i^{\prime}}\right] f_{1} T_{S}}\right\} \\
& \text { - }\left[M\left\{e^{-j 2 \pi\left[(u-k) Z_{w i}+\left(u^{\prime}-k\right) Z_{w i^{\prime}}\right] f_{1} T_{A}}\right\}\right. \\
& +M\left\{e^{-j 2 \pi\left[(u-k) Z_{w i}+\left(u^{\prime}+k\right) Z_{w i^{\prime}}\right] f_{1} T_{A}}\right\} \\
& +M\left\{e^{-j 2 \pi\left[(u+k) Z_{w i}+\left(u^{\prime}-k\right) Z_{w i^{\prime}}\right] f_{1} T_{A}}\right\} \\
& \left.+M\left\{e^{-j 2 \pi\left[(u+k) Z_{w i}+\left(u^{\prime}+k\right) Z_{w i i^{\prime}}\right] f_{1} T_{A}}\right\}\right] .
\end{aligned}
$$

$$
\begin{aligned}
& \underset{M}{M}\left\{\left|\tilde{X}_{k, w}\right|^{4}\right\}=\frac{1}{4 N^{2}} \sum_{r=-M}^{+M} \sum_{u=-M}^{+M} \sum_{r^{\prime}=-M}^{+M} \sum_{\substack{u^{\prime}=-M \\
r+u=-\left(r^{\prime}+u^{\prime}\right)}}^{+M} \\
& \cdot X_{r} X_{u} X_{r^{\prime}} X_{u^{\prime}} \sum_{i=0}^{N-1} \sum_{i^{\prime}=0}^{N-1} e^{-j 2 \pi(r+u)\left(i-i^{\prime}\right) f_{1} T_{S}} \\
& \cdot M\left\{e^{j 2 \pi(r+u)\left(Y_{w-i}-Y_{w-i^{\prime}}\right) f_{1} T_{S}}\right\} \\
& \text { - }\left[M\left\{e^{-j 2 \pi\left[(u-k) Z_{w i}+\left(u^{\prime}-k\right) Z_{w i^{\prime}}\right] f_{1} T_{A}}\right\}\right.
\end{aligned}
$$

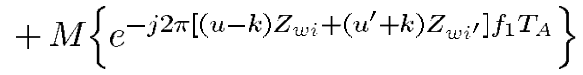

$$
\begin{aligned}
& +M\left\{e^{-j 2 \pi\left[(u+k) Z_{w i}+\left(u^{\prime}-k\right) Z_{w i^{\prime}}\right] f_{1} T_{A}}\right\} \\
& \left.+M\left\{e^{-j 2 \pi\left[(u+k) Z_{w i}+\left(u^{\prime}+k\right) Z_{w i^{\prime}}\right] f_{1} T_{A}}\right\}\right] .
\end{aligned}
$$

This mean value can be expressed as the sum of two terms $M_{1}$ and $M_{2}$ obtained by considering separately the contributions for $i=i^{\prime}$ and $i \neq i^{\prime}$ in the double sum with respect to $i$ and $i^{\prime}$. These two terms must be real because the variance is real in accordance to (A15). Thus, we can write

$$
\underset{\rightarrow}{M}\left\{\left|\tilde{X}_{k, w}\right|^{4}\right\}=M_{1}+M_{2}
$$

where

$$
\begin{aligned}
M_{1}=\frac{1}{4 N^{2}} \sum_{r=-M}^{+M} \sum_{u=-M}^{+M} \sum_{r^{\prime}=-M}^{+M} \sum_{\substack{u^{\prime}=-M \\
r+u=-\left(r^{\prime}+u^{\prime}\right)}}^{+M} X_{r} X_{u} \\
\cdot X_{r^{\prime}} X_{u^{\prime}} \sum_{i=0}^{N-1}\left[M \left\{e^{-j 2 \pi\left(u+u^{\prime}-2 k\right) Z_{w i} f_{1} T_{A}}\right.\right. \\
+2 M\left\{e^{-j 2 \pi\left(u+u^{\prime}\right) Z_{w i} f_{1} T_{A}}\right\} \\
\left.\left.+M\left\{e^{-j 2 \pi\left(u+u^{\prime}+2 k\right) Z_{w i} f_{1} T_{A}}\right\}\right\}\right]
\end{aligned}
$$

and, due to the independence of the involved random variables 
for $i \neq i^{\prime}$

$$
\begin{aligned}
M_{2}= & \frac{1}{4 N^{2}} \sum_{r=-M}^{+M} \sum_{u=-M}^{+M} \sum_{r^{\prime}=-M}^{+M} \sum_{\substack{u^{\prime}=-M \\
r+u=-\left(r^{\prime}+u^{\prime}\right)}}^{+M} \\
& \cdot X_{r} X_{u} X_{r^{\prime}} X_{u^{\prime}} \sum_{i=0}^{N-1} \sum_{\substack{i^{\prime}=0 \\
i \neq i^{\prime}}}^{N-1} e^{-j 2 \pi(r+u)\left(i-i^{\prime}\right) f_{1} T_{S}} \\
& \cdot M\left\{e^{j 2 \pi(r+u) Y_{w-i} f_{1} T_{S}}\right\} M\left\{e^{-j 2 \pi(r+u) Y_{w-i^{\prime}} f_{1} T_{S}}\right\} \\
& \cdot M\left\{e^{-j 2 \pi(u-k) Z_{w i} f_{1} T_{A}}\right\}\left(M\left\{e^{-j 2 \pi\left(u^{\prime}-k\right) Z_{w i^{\prime}} f_{1} T_{A}}\right\}\right. \\
& \left.+M\left\{e^{-j 2 \pi\left(u^{\prime}+k\right) Z_{w i^{\prime}} f_{1} T_{A}}\right\}\right)+M\left\{e^{-j 2 \pi(u+k) Z_{w i} f_{1} T_{A}}\right\} \\
& \cdot\left(M\left\{e^{-j 2 \pi\left(u^{\prime}-k\right) Z_{w i^{\prime}} f_{1} T_{A}}\right\}\right. \\
& \left.\left.+M\left\{e^{-j 2 \pi\left(u^{\prime}+k\right) Z_{w i^{\prime}} f_{1} T_{A}}\right\}\right)\right] .
\end{aligned}
$$

Now, let us remember that the variability range of the shift $\tau$ has been assumed synchronized with the input signal so that $f_{1} T_{A}=1$. Consequently, in order to have nonnull contributions to $M_{1}$ in (A21), in accordance to (A2) and (A7), the first term between brackets in (A21) impose that $u+u^{\prime}=$ $2 k$, the second one $u+u^{\prime}=0$, and the third $u+u^{\prime}=-2 k$. Therefore, since the condition $r+u=-\left(r^{\prime}+u^{\prime}\right)$ must always be satisfied, in the three cases we obtain, respectively, the following correspondences: $r+r^{\prime}=-2 k$ in the first case, $r+r^{\prime}=0$ in the second one, and $r+r^{\prime}=+2 k$ in the last one. Therefore, (A21) can be written as follows:

$$
\begin{aligned}
& M_{1}=\frac{1}{4 N}\left[\sum_{r=-M}^{+M} \sum_{u=-M}^{+M} \sum_{\begin{array}{c}
r^{\prime}=-M \\
u^{\prime}=-M \\
u+u^{\prime}=2 k \\
r+r^{\prime}=-2 k
\end{array}}^{+M} X_{r} X_{u} X_{r^{\prime}} X_{u^{\prime}}\right. \\
& +2 \sum_{r=-M}^{+M} \sum_{u=-M}^{+M} \sum_{r^{\prime}=-M}^{+M} \sum_{\begin{array}{c}
u^{\prime}=-M \\
u=-u^{\prime} \\
r=-r^{\prime}
\end{array}}^{+M} X_{r} X_{u} X_{r^{\prime}} X_{u^{\prime}} \\
& \left.+\sum_{r=-M}^{+M} \sum_{u=-M}^{+M} \sum_{r^{\prime}=-M}^{+M} \sum_{\begin{array}{r}
u^{\prime}=-M \\
u+u^{\prime}=-2 k \\
r+r^{\prime}=2 k
\end{array}}^{+M} X_{r} X_{u} X_{r^{\prime}} X_{u^{\prime}}\right] \\
& =\frac{1}{4 N}\left[\sum_{r=-M}^{+M} X_{r} X_{2 k+r}^{*} \sum_{u=-M}^{+M} X_{u} X_{2 k-u}\right. \\
& +2 \sum_{r=-M}^{+M} X_{r} X_{r}^{*} \sum_{u=-M}^{+M} X_{u} X_{u}^{*} \\
& \left.+\sum_{r=-M}^{+M} X_{r} X_{2 k-r} \sum_{u=-M}^{+M} X_{u} X_{2 k+u}^{*}\right]
\end{aligned}
$$

By changing in the last term $(r, u)$ into $(-r,-u)$ it results the complex conjugate of the first one

$$
\begin{aligned}
& M_{1}=\frac{1}{4 N}[ \sum_{r=-M}^{+M} X_{r} X_{2 k+r}^{*} \sum_{u=-M}^{+M} X_{u} X_{2 k-u} \\
&+2 \sum_{r=-M}^{+M} X_{r} X_{r}^{*} \sum_{u=-M}^{+M} X_{u} X_{u}^{*} \\
&\left.+\sum_{r=-M}^{+M} X_{r}^{*} X_{2 k+r} \sum_{u=-M}^{+M} X_{u}^{*} X_{2 k-u}^{*}\right] \\
&=\frac{1}{2 N}\left\{\left(\sum_{r=-M}^{+M}\left|X_{r}^{2}\right|\right)^{2}+\left|\sum_{r=-M}^{+M} X_{r} X_{2 k-r}\right|^{2}\right\} .
\end{aligned}
$$

Therefore, the quantity $M_{1}$ is real as provided by (15).

Now, by considering $M_{2}$ of (A22), in analogy with the previous treatment regarding $M_{1}$, we can deduce that the first product between brackets gives nonnull contributions only for $u=u^{\prime}= \pm k$, the second one for $u=-u^{\prime}=k$, the third one for $u=-u^{\prime}=-k$, the last one for $u=u^{\prime}=-k$. Since the condition $r+u=-\left(r^{\prime}+u^{\prime}\right)$ and thus $r+r^{\prime}=-\left(u+u^{\prime}\right)$ must always be satisfied, in the four cases we obtain the following correspondences:

$$
\begin{aligned}
& u=u^{\prime}=k \rightarrow r+r^{\prime}=-2 k \\
& u=-u^{\prime}=k \rightarrow r+r^{\prime}=0 \\
& u=-u^{\prime}=-k \rightarrow r+r^{\prime}=0 \\
& u=u^{\prime}=-k \rightarrow r+r^{\prime}=2 k .
\end{aligned}
$$

Thus, (A22) can be rewritten as follows:

$$
\begin{aligned}
M_{2}= & \frac{1}{4 N^{2}} \sum_{r=-M}^{+M}\left[X_{r} X_{k}^{2} X_{2 k+r}^{*}+\left|X_{r}\right|^{2}\left|X_{k}\right|^{2}\right] \\
& \cdot \sum_{i=0}^{N-1} \sum_{\substack{i^{\prime}=0 \\
i \neq i^{\prime}}}^{N-1} e^{-j 2 \pi(r+k)\left(i-i^{\prime}\right) f_{1} T_{S}} \\
& \cdot M\left\{e^{j 2 \pi(r+k) Y_{w-i} f_{1} T_{S}}\right\} M\left\{e^{-j 2 \pi(r+k) Y_{w-i^{\prime}} f_{1} T_{S}}\right\} \\
& +\frac{1}{4 N^{2}} \sum_{r=-M}^{+M}\left[\left|X_{r}\right|^{2}\left|X_{k}\right|^{2}+X_{r}\left(X_{k}^{*}\right)^{2} X_{2 k-r}\right] \\
& \cdot \sum_{i=0}^{N-1} \sum_{\substack{i^{\prime}=0 \\
i \neq i^{\prime}}}^{N-1} e^{-j 2 \pi(r-k)\left(i-i^{\prime}\right) f_{1} T_{S}} \\
& \cdot M\left\{e^{j 2 \pi(r-k) Y_{w-i} f_{1} T_{S}}\right\} M\left\{e^{-j 2 \pi(r-k) Y_{w-i^{\prime}} f_{1} T_{S}}\right\} .
\end{aligned}
$$


We observe that changing $r$ into $-r$ in the second term of the sum, it results the complex conjugate of the first one. Thus also $M_{2}$ is real. By taking into account (A2), we obtain

$$
\begin{aligned}
M_{2}= & \frac{1}{2 N^{2}} \sum_{r=-M}^{+M} \operatorname{sinc}^{2}(r+k) f_{1} T_{s} \\
& \cdot\left\{\operatorname{Re}\left[X_{r} X_{k}^{2} X_{2 k+r}^{*}\right]+\left|X_{r}\right|^{2}\left|X_{k}\right|^{2}\right\} \\
& \cdot \sum_{i=0}^{N-1} \sum_{\substack{i^{\prime}=0 \\
i \neq i^{\prime}}}^{N-1} e^{-j 2 \pi(r+k)\left(i-i^{\prime}\right) f_{1} T_{S}} .
\end{aligned}
$$

By recalling that we can always write

$$
\sum_{i=0}^{N-1} \sum_{\substack{i^{\prime}=0 \\ i \neq i^{\prime}}}^{N-1}(\cdot)=\sum_{i=0}^{N-1} \sum_{i^{\prime}=0}^{N-1}(\cdot)-\sum_{i=0}^{N-1}(\cdot)
$$

it follows from (A5) that the double sum of (A26) can be rewritten as follows:

$$
\begin{aligned}
& \sum_{i=0}^{N-1} \sum_{\substack{i^{\prime}=0 \\
i \neq i^{\prime}}}^{N-1} e^{-j 2 \pi(r+k)\left(i-i^{\prime}\right) f_{1} T_{S}} \\
& =\sum_{i=0}^{N-1} e^{-j 2 \pi(r+k) i f_{1} T_{S}} \sum_{i^{\prime}=0}^{N-1} e^{j 2 \pi(r+k) i^{\prime} f_{1} T_{S}}-N \\
& =N^{2} \frac{\operatorname{sinc}^{2}\left((r+k) N f_{1} T_{S}\right)}{\operatorname{sinc}^{2}\left((r+k) f_{1} T_{S}\right)}-N .
\end{aligned}
$$

By substituting into (A26), we obtain

$$
\begin{aligned}
M_{2}= & \frac{1}{2 N^{2}} \sum_{r=-M}^{+M} \operatorname{sinc}^{2}(r+k) f_{1} T_{s} \\
& \cdot\left\{\operatorname{Re}\left[X_{r} X_{k}^{2} X_{2 k+r}^{*}\right]+\left|X_{r}\right|^{2}\left|X_{k}\right|^{2}\right\} \\
& \cdot\left[\frac{\operatorname{sinc}^{2}\left((r+k) N f_{1} T_{S}\right)}{\operatorname{sinc}^{2}\left((r+k) f_{1} T_{S}\right)}-\frac{1}{N}\right] .
\end{aligned}
$$

The expressions of $M_{1}$ (A24) and $M_{2}$ (A29) can now be introduced into (A20)

$$
\begin{aligned}
& \stackrel{M}{\rightarrow}\left\{\left|\tilde{X}_{k, w}\right|^{4}\right\} \\
& =\frac{1}{2 N}\left[\left(\sum_{r=-M}^{+M}\left|X_{r}\right|^{2}\right)^{2}+\left|\sum_{r=-M}^{+M} X_{r} X_{2 k-r}\right|^{2}\right] \\
& \quad+\frac{1}{2} \sum_{r=-M}^{+M} \operatorname{Re}\left[X_{r} X_{k}^{2} X_{2 k+r}^{*}+\left|X_{r}\right|^{2}\left|X_{k}\right|^{2}\right] \\
& \quad \cdot \operatorname{sinc}^{2}\left((r+k) N f_{1} T_{S}\right)\left(\frac{\operatorname{sinc}^{2}\left((r+k) N f_{1} T_{S}\right)}{\operatorname{sinc}^{2}\left((r+k) f_{1} T_{S}\right)}-\frac{1}{N}\right)
\end{aligned}
$$

and, by referring to (A15), we can deduce the final expression of the asymptotic variance

$$
\begin{aligned}
& \underset{\operatorname{Var}}{\rightarrow}\left\{\left|\tilde{X}_{k, w}\right|^{2}\right\} \\
& \quad=\frac{1}{2 N}\left[\left(\sum_{r=-M}^{+M}\left|X_{r}\right|^{2}\right)^{2}+\left|\sum_{r=-M}^{+M} X_{r} X_{2 k-r}\right|^{2}\right]
\end{aligned}
$$

$$
\begin{aligned}
& +\frac{1}{2} \sum_{r=-M}^{+M}\left\{\operatorname{Re}\left[X_{r} X_{k}^{2} X_{2 k+r}^{*}\right]+\left|X_{r}\right|^{2}\left|X_{k}\right|^{2}\right\} \\
& \cdot \operatorname{sinc}^{2}\left((r+k) f_{1} T_{S}\right)\left(\frac{\operatorname{sinc}^{2}\left((r+k) N f_{1} T_{S}\right)}{\operatorname{sinc}^{2}\left((r+k) f_{1} T_{S}\right)}-\frac{1}{N}\right) \\
& -\left|X_{k}\right|^{4} .
\end{aligned}
$$

\section{REFERENCES}

[1] G. Iuculano, D. Mirri, F. Filicori, A. Menchetti, and M. Catelani, "A criterion for the performance analysis of synchronous and asynchronous sampling instruments based on non linear processing," in Inst. Elec. Eng. Proc., vol. 139, no. 4, pt. A, pp. 141-152, July 1992.

[2] D. Mirri, G. Iuculano, A. Menchetti, F. Filicori, and M. Catelani, "Recursive random sampling strategy for a digital wattmeter," IEEE Trans. Instrum. Meas., vol. 41, pp. 979-984, Dec. 1992.

[3] E. O. Brigham, The Fast Fourier Transform and its Applications. Englewood Cliffs, NJ: Prentice Hall International, 1988.

[4] W. McC. Siebert, Circuits, Signals, and Systems. New York: McGrawHill, 1986.

[5] J. M. Hammersley and D. C. Handscomb, Montecarlo Methods. London, U.K.: Chapman and Hall, 1964.

[6] W. Feller, An Introduction to Probability Theory and Its Applications. New York: Wiley, 1971.

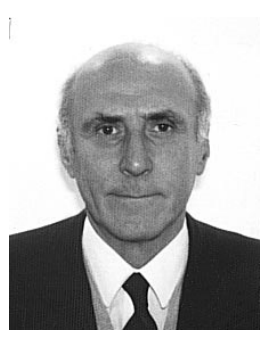

Domenico Mirri (M'91) was born in Italy in 1936. $\mathrm{He}$ received the electronic engineering degree from the University of Bologna, Bologna, Italy.

Presently, he is an Associate Professor of Electronic Measurement at the University of Bologna. His current research interest is in the areas of digital measurement instruments, devices metrological characterization, and biomedical measurements.

Gaetano Iuculano was born in Italy in 1938. He received the degree in electronic engineering from the University of Bologna, Bologna, Italy.

Presently, he is a Full Professor of Electrical Measurements at the University of Florence, Italy. His current research interests are in calibration applications, reliability analysis and life testing for electronic devices and systems, statistical analysis, and digital measurement instruments.

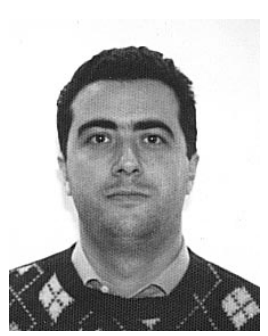

Gaetano Pasini (M'97) was born in Italy in 1964. $\mathrm{He}$ received the degree in electronic engineering from the University of Bologna, Bologna, Italy.

$\mathrm{He}$ is currently a Researcher in electrical measurement. His research activity is mainly oriented to digital signal processing in electronic instruments, power measurements, and characterization on nonlinear systems with memory.

Fabio Filicori (M'98) was born in Italy in 1949. He received the degree in electronic engineering from the University of Bologna, Bologna, Italy, in 1964.

Presently, he is a Full Professor of Electrical Measurements at the University of Bologna. His current research interests are in the areas of nonlinear circuit analysis and design, electronic device modeling, digital measurement instruments, and power electronics.

Lorenzo Peretto (M'98) was born in Italy in 1968. He received the degree in electronic engineering from the University of Bologna, Bologna, Italy. He is currently working towards the Ph.D. degree in the Department of Electrical Engineering at the University of Bologna.

His actual field of interest is the measurement in power systems under nonsinusoidal conditions. 